\title{
Detuning Control of Resonant Wireless Energy Transmission System Based on CGI-PLL
}

\author{
Peicong Qian*, Jinzhi Wang, Xuan Zhang \\ College of Electrical Engineering, Guangxi University, Nanning, China \\ Email address: \\ 2810468787@qq.com (Peicong Qian),625948165@qq.com (Jinzhi Wang),465957051@qq.com (Xuan Zhang) \\ ${ }^{*}$ Corresponding author
}

To cite this article:

Peicong Qian, Jinzhi Wang, Xuan Zhang. Detuning Control of Resonant Wireless Energy Transmission System Based on CGI-PLL. Journal of Electrical and Electronic Engineering. Vol. 7, No. 6, 2019, pp. 143-150. doi: 10.11648/j.jeee.20190706.12

Received: November 4, 2019; Accepted: November 22, 2019; Published: December 3, 2019

\begin{abstract}
At present, in the practical application of magnetic resonance wireless transmission technology, because of the change of distance, relative position between transmitting coil and receiving coil and load, the output power and transmission efficiency of transmission system will be greatly affected. Therefore, in order to improve the output power and transmission efficiency. In this paper, a new frequency tracking control algorithm based on Cascaded Generalized Integrator PLL (CGI-PLL) structure is proposed. The new frequency tracking control algorithm is used in the series- series circuit of magnetic resonance. It is planned to use this algorithm when the transmitting coil is detuning to a certain extent and the receiving coil is resonant at the same time. The phase compensation and soft switch are realized by controlling the phase difference between the output voltage and the output current of the primary side inverter. The experimental results show that the phase between the output voltage and the output current of the primary measurement inverter can remain unchanged when the load and mutual inductance are changed. Therefore, the CGI-PLL detuning control algorithm can actively track the frequency of the system, improve the output power and transmission efficiency of the system, and provide conditions for the operation of ZVS soft switch. It is proved that the new control algorithm proposed in this paper is feasible and correct.
\end{abstract}

Keywords: Wireless Energy, Resonant Circuit, PLL, ZVS

\section{Introduction}

The magnetic resonance wireless energy transmission system has a longtransmission distance and high transmission efficiency, which has attracted the attention of many scholars at home and abroad [1-3]. However, the magnetic resonance wirelessenergy transmission technology is sensitive to frequency conversion $[4,5]$. This technology requires that the resonance frequency of the transmitting coil and the receiving coil is the same [6, 7]. In addition, load change and transmission distance change and other factors may cause system detuning [8, 9]. In reference [10], an adaptive tuning method of the magnetic amplifier is adopted, which tracks the phase difference between the power supply and the load voltage, and adjusts the controllable inductance at the same time. In view of the low transmission efficiency caused by the detuning, there are two main methods at home and abroad: one is adaptive impedance control, the other is passive frequency tracking control. In reference [11], by changing the connection mode of series and parallel capacitance matrix, the optimal capacitance combination of the system can be obtained, but this method will cause large delay and make the system unstable. In [12], a controllable capacitor array is added to change the value of compensation capacitance at the transmitter. The accuracy of the impedance adaptive method is determined by the number of compensation capacitance, which increases the difficulty of hardware circuit design. In reference [13], a zero-crossing detection phase-locked loop is introduced to provide conditions for the system to be in a resonant state, but this method is susceptible to the interference of input abnormal signals. In reference [14], the frequency tracking method of short-circuit current detection is introduced. This method can realize frequency tracking quickly, but the system is vulnerable to external interference, which will produce some errors. At present, the main methods of frequency tracking based on PLL are: $\mathrm{T} / 4$ delay PLL, anti-Park transform adaptive filter, and differential PLL frequency tracking method $[15,16]$.

In paper [17] generalized second order integral phase 
locked loop (SOGI-PLL) method is adopted to control detuning control and obtain good control effect. Based on literature [17], this paper adopts cascaded generalized integrator phase locked loop (CGI-PLL) [18] structure to filter input signals effectively and track frequency of input signals so as to improve precision and adaptive capability of PLL frequency. 2. Model and Analysis of Magnetic Wireless Energy Transmission System

\section{Model and Analysis of Magnetic Wireless Energy Transmission System}

As shown in Figure 1 [17], the system consists of DC power, inverter, resonant transmitting coil, receiving coil, rectifier and load. According to the principle of coupled equivalent circuit, the Figure 1 can be simplified into Figure 2.

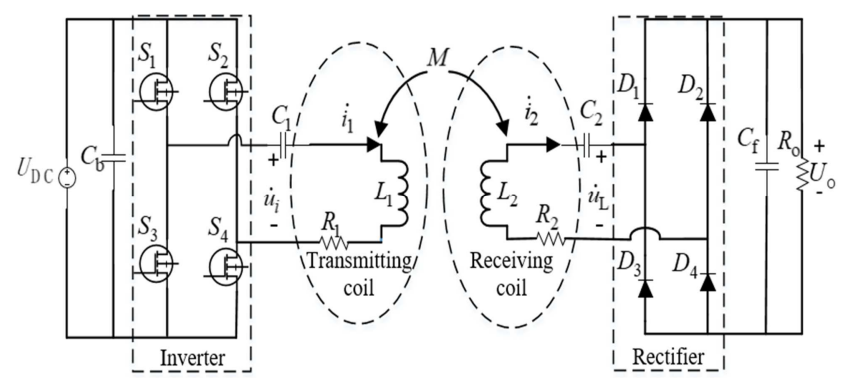

Figure 1. Circuit of series magnetic resonance power transmission.

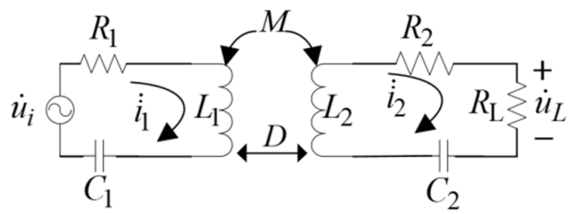

Figure 2. Seriesmagnetic resonance power transfer equivalent circuit.

$D$ is the distance from the transmitting coil to the receiving coil, and $Z_{1}$ and $Z_{2}$ are the equivalent impedance of the transmitting coil and the receiving coil is the angular frequency of the system; $i_{1}$ and $i_{2}$ are the current of the transmitting coil and the receiving coil. From Figure 2, we can deduce the equation (1):

$$
\left\{\begin{array}{c}
\dot{i}_{1}=Z_{2} \dot{u}_{i} /\left(Z_{1} Z_{2}+(\omega M)^{2}\right) \\
\dot{i}_{2}=\mathrm{j} \omega M \dot{u}_{i} /\left(Z_{1} Z_{2}+(\omega M)^{2}\right)
\end{array}\right.
$$

we can get input power $P_{\mathrm{i}}$ and output power $P_{\mathrm{o}}$ of system. As (2)

$$
\left\{\begin{array}{c}
P_{\mathrm{i}}=\left|\dot{u}_{i}\right|^{2} Z_{2} /\left(Z_{1} Z_{2}+(\omega M)^{2}\right)^{2} \\
P_{\mathrm{o}}=\left|\dot{u}_{i}\right|^{2}(\omega M)^{2} R_{\mathrm{L}} /\left(Z_{1} Z_{2}+(\omega M)^{2}\right)^{2}
\end{array}\right.
$$

Form (2), the transmission efficiency between the transmitting coil and the receiving coil is:

$$
\eta=(\omega M)^{2} R_{\mathrm{L}} /\left[Z_{2}\left(Z_{1} Z_{2}+(\omega M)^{2}\right)\right] \cdot 100 \%
$$

It can be seen from (1) to (3) that the transmission efficiency and output power of the system are related to system frequency, load, mutual inductance, resonance capacitance, resonance inductance and other factors.

\section{Detuning Control Strategy of Wireless Energy Transmission System Based on CGI-PLL}

The control strategy is an active frequency tracking compensation strategy. As shown in Figure 3, the control system is composed of the main circuit module of the magnetic resonance wireless energy transmission system, the CGI-PLL control circuit module, the pulse modulated PFM signal generator and the driving circuit.

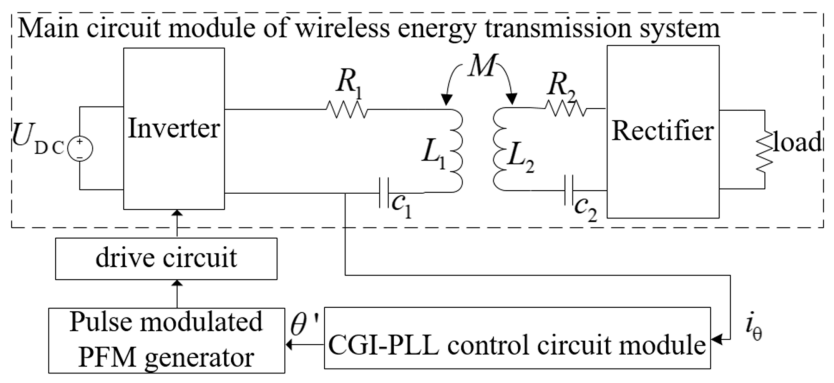

Figure 3. CGI-PLL magnetic resonance power transmission system.

The implementation process of the detuning control algorithm is shown in Figure 4:

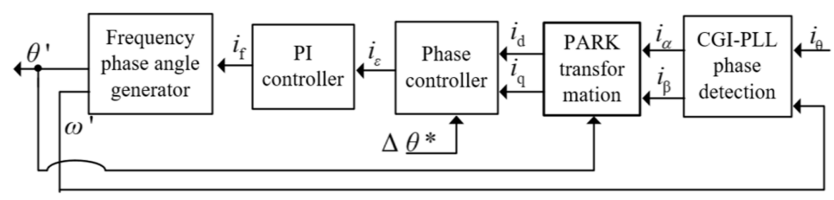

Figure 4. CGI-PLL detuning control algorithm block diagram.

\subsection{CGI-PLLPhase Detection Module}

CGI-PLL phase detector is a kind of orthogonal signal generator based on adaptive filter. Its main function is to decompose input signal $i_{\theta}$ into two orthogonal signals $i_{\alpha}$ and $i_{\beta}$. It can process signals with large frequency range and has strong anti-interference ability. Its structure is shown in Figure 5 [18].

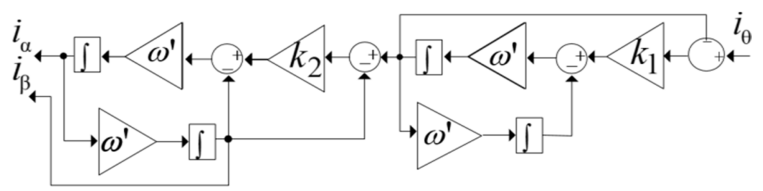

Figure 5. Phase detector loop of CGI-PLL.

When a wave form $i_{\theta}$ input to system:

$$
i_{\theta}=\sum_{\mathrm{n}=1}^{\mathrm{m}} i_{\mathrm{n}} \sin \left(\mathrm{n} \omega t+\varphi_{\mathrm{n}}\right)
$$

Above, $i_{\mathrm{n}}, \mathrm{n} \omega$ and $\varphi_{\mathrm{n}}$ respectively represent the amplitude, 
angular frequency and initial phase of the $i_{\theta}$. When $\mathrm{n}=1$, the input signal frequency is equal to the center frequency of CGI-PLL, and the amplitude of the output signal is equal to that of the output signal. When $\mathrm{n} \neq 1$, the output signal frequency deviates from the center frequency of CGI-PLL, and the output amplitude decreases. The output signal $i_{\alpha}$ and $i_{\beta}$ maintain a fixed $90^{\circ}$ phase difference, that is, the two output signals are orthogonal. When $k_{1}=k_{2}=1.63$ [18], the amplitude frequency and phase frequency characteristic curve of CGI-PLL phase detection ring is shown in Figure 6.

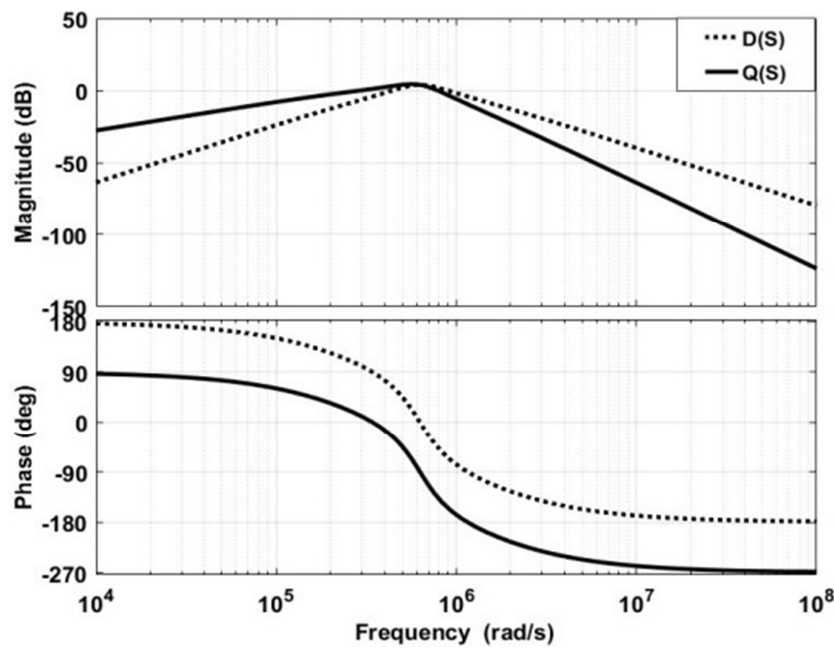

Figure 6. Bode plot of CGI-PLL phase-detection loop.

It can be seen from Figure 6 that $D(S)$ has band-pass characteristics, $Q(S)$ has low-pass filtering characteristics, and the bandwidth of the closed-loop system depends on the size of $k_{1}$ and $k_{2}$, and affects the transient response and harmonic attenuation ability of the system.

\subsection{PARK Transformation}

The main function of Park transformation is to transform two-phase rotating coordinates into two-phase static coordinates. $i_{\alpha}$ and $i_{\beta}$ are converted into $i_{\mathrm{d}}$ and $i_{\mathrm{q}}$, and the transformation relationship between the two-phase static coordinate system and the rotating coordinate system, i.e. Park transformation, is shown in Figure 7 . Where, $\theta$ is the phase angle of input signal and $\theta^{\prime}$ is the phase angle of CGI-PLL output signal [17].

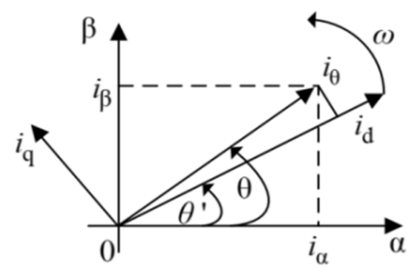

Figure 7. Coordinate of PARK transformation.

According to Figure 7, $i_{\mathrm{d}}$ and $i_{\mathrm{q}}$ can be expressed as [17]:

$$
\left[\begin{array}{l}
i_{\mathrm{d}} \\
i_{\mathrm{q}}
\end{array}\right]=\left[\begin{array}{cc}
\cos \theta^{\prime} & \sin \theta^{\prime} \\
-\sin \theta^{\prime} & \cos \theta^{\prime}
\end{array}\right]\left[\begin{array}{l}
i_{\alpha} \\
i_{\beta}
\end{array}\right]
$$

Therefore, it can be considered that $i_{\mathrm{q}}$ can represent the phase difference between input phase $\theta$ and output phase $\theta^{\prime}$, and can reflect the synchronization between $d$ axis and input signal.

\subsection{Phase Control}

In order to ensure that the system work in the primary coil detuning and the secondary coil resonance. The phase angle difference controller is designed to control the phase difference between CGI-PLL output signal and output signal accurately. Figure 8 shows the phase control process [17].

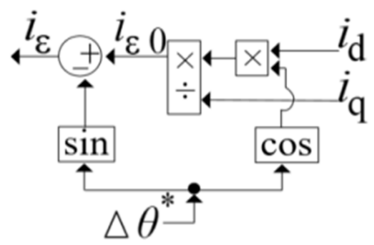

Figure 8. Phase control.

In Figure 8, The introduced intermediate variable is expressed as [17]:

$$
\begin{aligned}
& i_{\varepsilon 0}=\sin \left(\Delta \theta^{*}\right) \\
& \Delta \theta=\theta^{\prime}-\theta=\Delta \theta^{*}
\end{aligned}
$$

Therefore, the phase difference control of CGI-PLL input signal and output signal can be realized through the control amount $\Delta \theta^{*}$.

\subsection{Frequency/Phase Angle Generator}

The function of frequency phase angle generator is to calculate the output signal $i_{\mathrm{f}}$ of PI controller and generate phase angle $\theta^{\prime}$ and angle frequency $\omega^{\prime}$. Its structure is shown in Figure 9 [17].

$$
\theta^{\prime} \frac{1}{s} \underset{\omega_{0}}{\omega^{\prime} \Delta \omega} k_{\mathrm{v} \mathrm{coo}} i_{\mathrm{f}}
$$

Figure 9. Frequency phase generator.

From Figure 9, it can be concluded that:

$$
\omega^{\prime}=\omega_{0}-\square \omega=\omega_{0}-k_{\mathrm{vco}} i_{\mathrm{f}}
$$

Where $\theta^{\prime}$ is the output of the frequency phase angle generator and provides the phase angle for the PARK transformation as the feedback; $\omega_{0}$ Represents the center angle frequency of CGI-PLL; $\omega^{\prime}$ is the output of the frequency phase angle generator and provides the angle frequency for CGI-PLL as the feedback; $\Delta \omega$ represents angular frequency compensation, which is used to indicate the degree of deviation between the frequency of input signal and center frequency. Gain factor $k_{\text {vco }}$ is used to adjust the accuracy range of frequency compensation.

\subsection{Stability Analysis for CGI-PLL}

Simplify Figure 4, and the simplified model is shown in 
Figure 10. CGI-PLL is composed of Phase Analyzer (PA), PI controller and Frequency/Phase Generator (FPG). The Phase Analyzer (PA) includes phase detection module, PARK transformation and phase control [17].

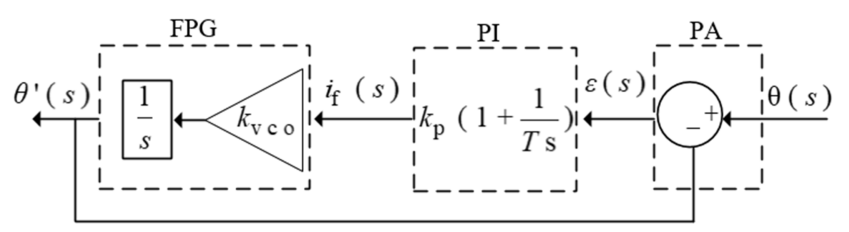

Figure 10. Simplified model for CGI-PLL.

Where $k_{\mathrm{p}}$ is the proportional gain coefficient regulated by $\mathrm{PI}$ and $\mathrm{T}$ is the integral time constant. The natural oscillation frequency of the system is: $\omega_{\mathrm{n}}=\sqrt{k_{\mathrm{p}} k_{\mathrm{vco}} / \text { T }}$ and the damping coefficient is: $\xi=\sqrt{\mathrm{k}_{\mathrm{p}} \mathrm{k}_{\mathrm{vco}}} / 2$. The damping coefficient is $\xi=0.7071$, and the noise bandwidth $\mathrm{B}_{\mathrm{L}}$ of CGI-PLL is: $B_{\mathrm{L}}=100000 \pi \mathrm{rad} / \mathrm{s}$. It can be concluded that: $\omega \approx 197461.46$. Above, we can draw the Bode diagram of CGI-PLL closed-loop system, that is, Figure 11.

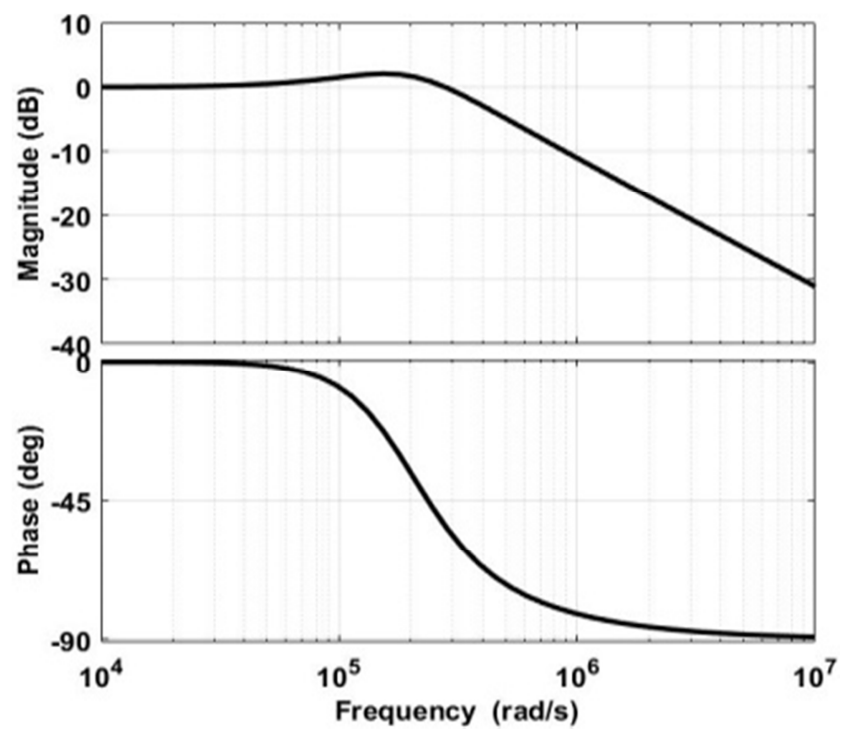

Figure 11. Bode plot of CGI-PLL closed-loop system.

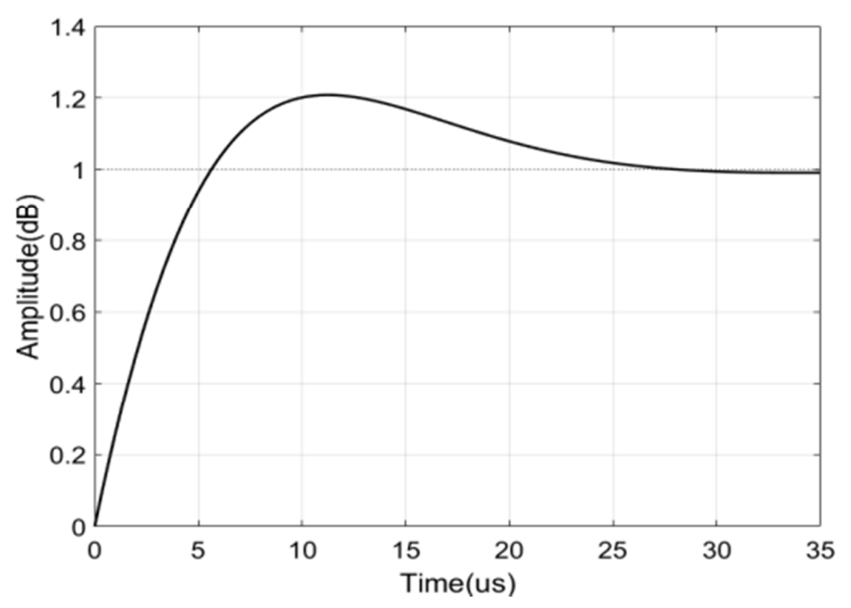

Figure 12. Step response curve of CGI-PLL.
It can be seen from Figure 11 that the gain of CGI-PLL closed-loop system is close to zero at low frequency, and the peak value is $2.1 \mathrm{~dB}$ at the resonance point of the system. Figure 12 is the step response curve of the closed-loop system. It can be considered that CGI-PLL system has good dynamic and static characteristics and is a stable and reliable system.

\section{Validation and Result Analysis}

In order to verify the feasibility of the proposed method, a simulation system is built for verification, and the designed circuit parameters are given in Table 1.

Table 1. Circuit parameters of CGI-PLL system.

\begin{tabular}{ll}
\hline Variable & Value \\
\hline $\mathrm{U}_{\mathrm{DC}} / \mathrm{V}$ & 60 \\
$\mathrm{C}_{1} / \mathrm{nF}$ & 620 \\
$\mathrm{C}_{2} / \mathrm{nF}$ & 620 \\
$\mathrm{~L}_{1} / \mu \mathrm{H}$ & 408.25 \\
$\mathrm{~L}_{2} / \mu \mathrm{H}$ & 408.25 \\
$\mathrm{R}_{1} / \Omega$ & 0.8 \\
$\mathrm{R}_{2} / \Omega$ & 0.8 \\
$\mathrm{f} / \mathrm{KHz}$ & 100 \\
\hline
\end{tabular}

In the practical application of the magnetic resonance wireless energy transmission system, there are many factors that lead to the system detuning, the most common one is the system detuning caused by the change of mutual inductance $M$ and load. The following two factors are analyzed.

\subsection{Effect of Mutual Inductance (M)}

In the actual system, the change of distance and relative position between the primary coil and the secondary coil will lead to the change of mutual inductance $(M)$, which will cause the system detuning. When the load $R_{\mathrm{o}}$ is $30 \Omega$, open-loop and closed-loop experiments are carried out with different mutual inductance $(M=20 \mu \mathrm{H}, 40 \mu \mathrm{H}, 60 \mu \mathrm{H}, 80 \mu \mathrm{H}, 100 \mu \mathrm{H}, 120$ $\mu \mathrm{H})$. Measure the output voltage $u_{\mathrm{i}}$ and output current $i_{1}$ of the primary end of the inverter. Figure 13 shows the open-loop waveform, and Figure 14 shows the closed-loop waveform.

From Figure 13 to Figure 14, in the absence of detuning control, there is a significant impedance angle between the output voltage and the output current of the primary inverter, and it changes significantly with the change of mutual inductance $M$. That is to say, the primary coil is in the detuning state, and the output current and voltage of the primary coil are no longer synchronized, which is not conducive to the operation of the ZVS. Figure 14 after CGI-PLL detuning control algorithm is adopted, the phase angle difference between $u_{\mathrm{i}}$ and $i_{1}$ can be controlled by controlling the size of input parameters $\Delta \theta^{*}$. Set $\Delta \theta^{*}=0.04 \pi$ here. After adopting CGI-PLL detuning control algorithm, with the change of mutual inductance $M, u_{\mathrm{i}}$ and $i_{1}$ are synchronized, and the phase angle between them remains unchanged, which provides conditions for the operation of ZVS. To further verify the effect of CGI-PLL control algorithm, the output power curve and transmission efficiency curve are drawn, as shown in Figure 15 and Figure 16. 


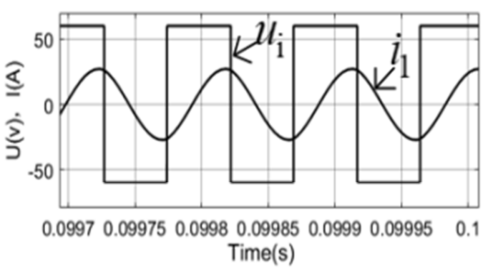

( a ). $M=20 \mu \mathrm{H}$

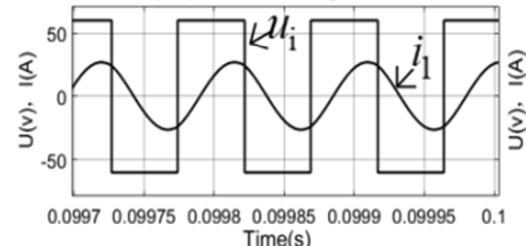

(c) $. M=60 \mu \mathrm{H}$

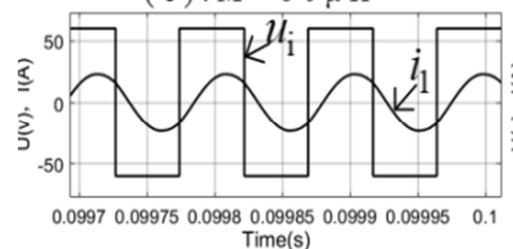

(e ). $M=100 \mu \mathrm{H}$

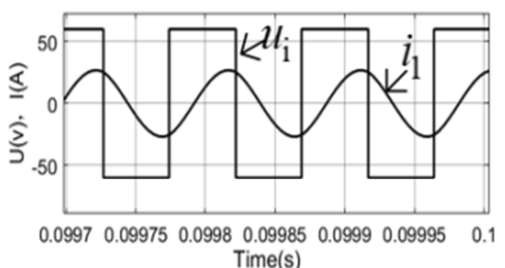

(b) $. M=40 \mu \mathrm{H}$

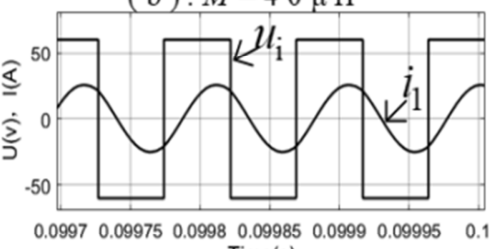

Time(s)

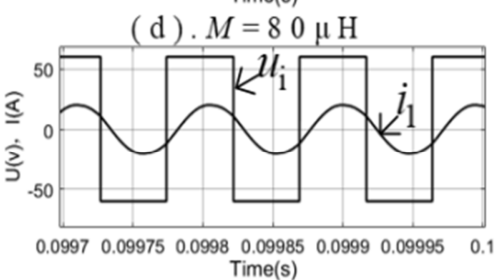

(f). $M=120 \mu \mathrm{H}$

Figure 13. Open-loop experimental of mutual inductance change.

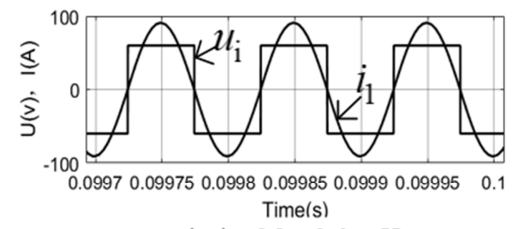

(a) $. M=20 \mu \mathrm{H}$

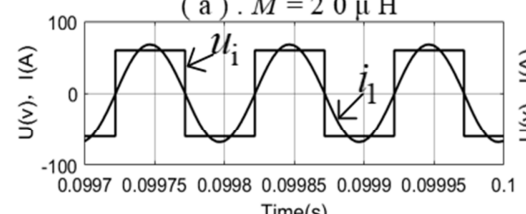

(c) $. M=60 \mu \mathrm{H}$

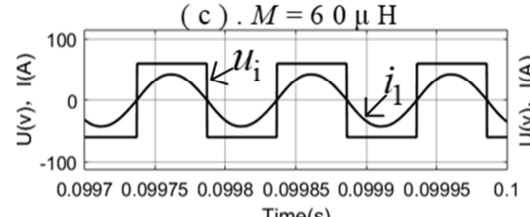

(e) $. M=100 \mu H$

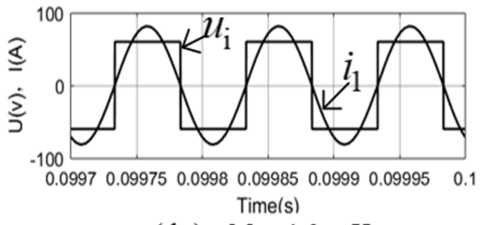

(b) $. M=40 \mu \mathrm{H}$

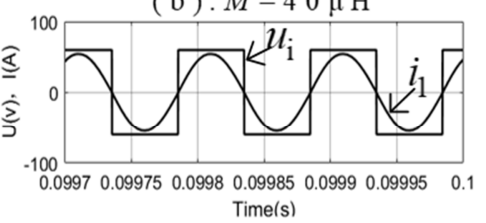

(d) $. M=80 \mu \mathrm{H}$

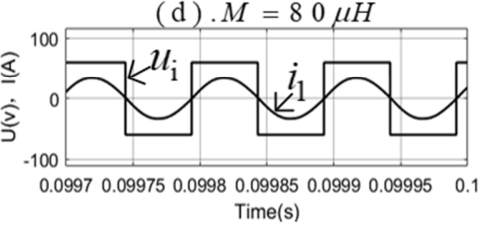

(f). $M=120 \mu \mathrm{H}$

Figure 14. Closed loop experiment of mutual inductance change.

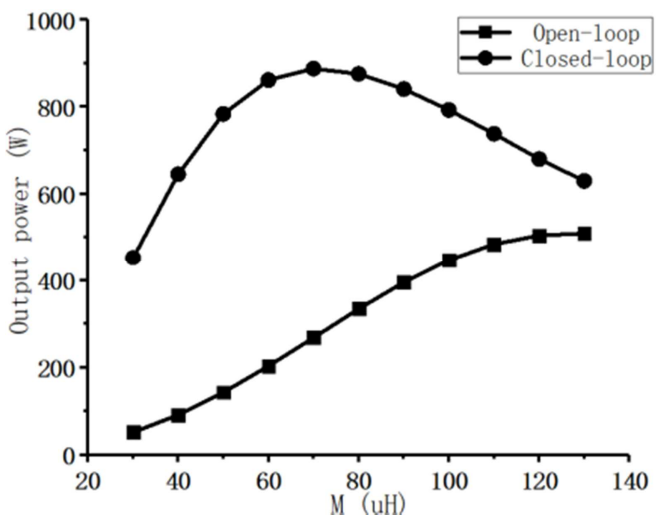

Figure 15. Relation of output power and mutual inductance.

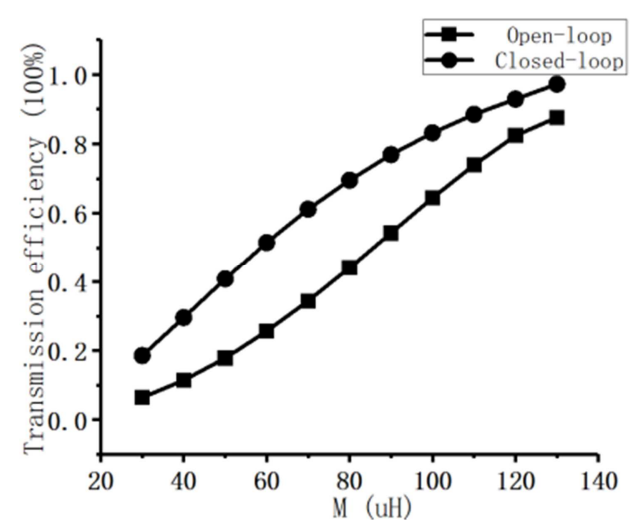

Figure 16. Relation of transmission efficiency and mutual inductance. 


\subsection{Effect of Load}

Under the condition of mutual inductance $M=100 \mu \mathrm{H}$ and different load resistances $\left(R_{0}=20 \Omega, 30 \Omega, 40 \Omega, 50 \Omega, 60 \Omega\right.$, $70 \Omega$ ). The following, open-loop and closed-loop comparative experiments are carried out. Measure the waveform of output voltage $u_{\mathrm{i}}$ and output current $i_{1}$ of the primary inverter. Figure 17 shows the waveform before the control algorithm is not used, and Figure 18 shows the waveform after the control algorithm is used.
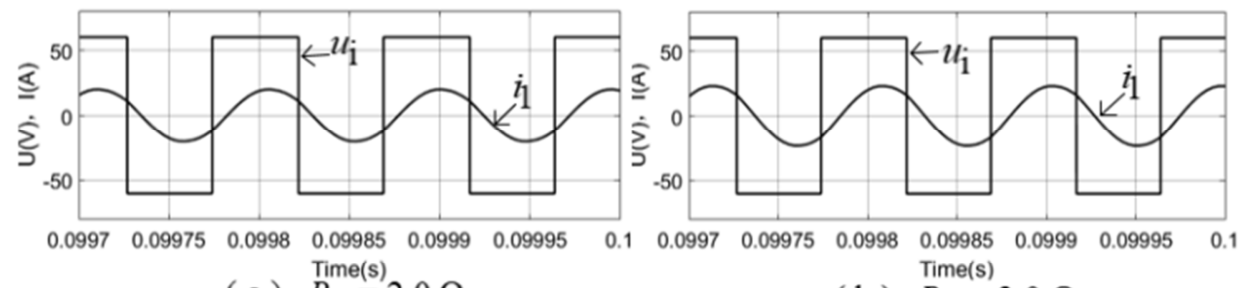

( a ).$R_{0} \stackrel{\text { Iimes }}{=} 20 \Omega$

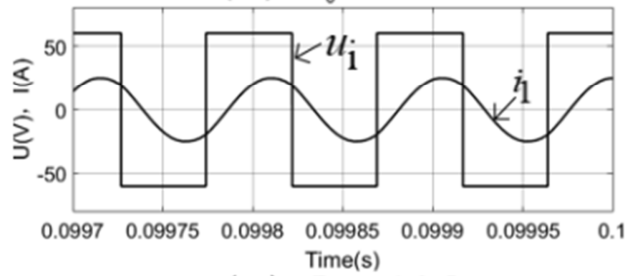

(b) $. R_{0}=30 \Omega$

(c) $. R_{0}=40 \Omega$

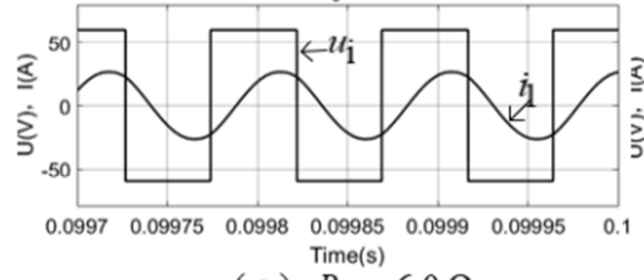

(e) $. R_{0}=60 \Omega$

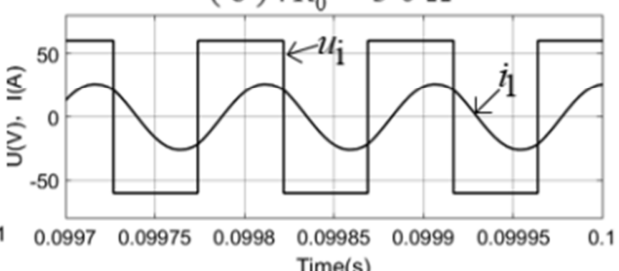

(d). $R_{0}=50 \Omega$

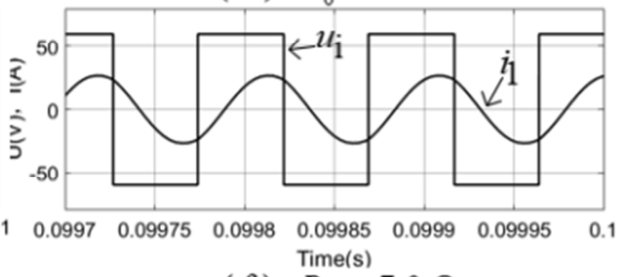

(f). $R_{0}=70 \Omega$

Figure 17. Open-loop experiment diagram of load change.

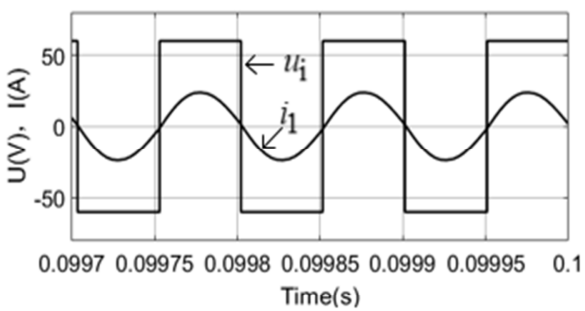

(a) $\cdot R_{0}=20 \Omega$

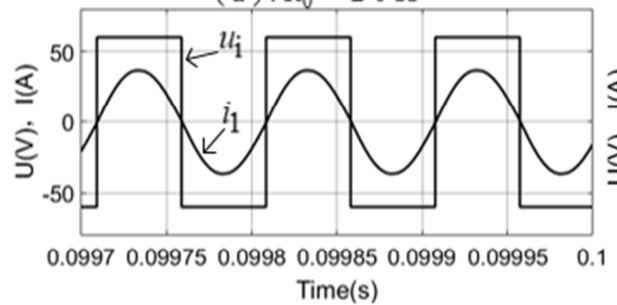

(c) $\cdot R_{0}=40 \Omega$

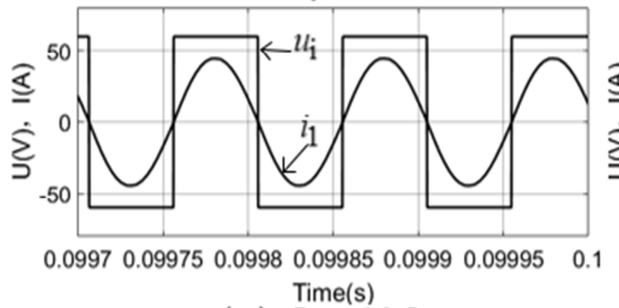

(e) $. R_{0}=60 \Omega$

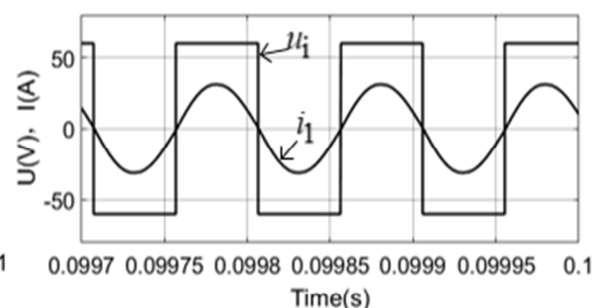

(b) $. R_{0}=30 \Omega$

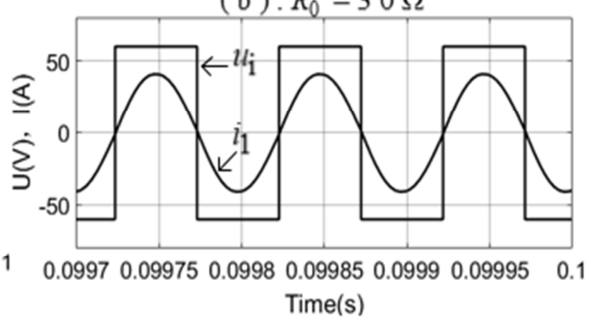

(d). $R_{0}=50 \Omega$

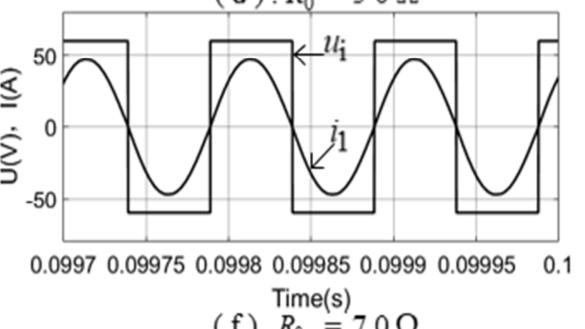

(f).$R_{0} \stackrel{(s)}{=} 70 \Omega$

Figure 18. Close-loop experiment diagram of load change. 
Figure 17 in the absence of control algorithm, there is an obvious impedance angle between the output voltage $u_{\mathrm{i}}$ and the output current $i_{1}$ of the primary inverter. And with the change of load, it changes obviously, that is to say, the primary terminal is in the state of detuning, and the output current and voltage of the primary terminal are no longer synchronized. Figure 18 shows the waveform after using CGI-PLL detuning control algorithm. Set the phase angle difference of $\Delta \theta^{*}=$ $0.04 \pi$. As the load changes, the phase angle between $u_{\mathrm{i}}$ and $i_{1}$ remains unchanged. That is to say, $u_{\mathrm{i}}$ and $i_{1}$ operate synchronously, which provides conditions for soft switch operation. To further verify the effect of CGI-PLL control algorithm, the output power curve and transmission efficiency curve are drawn, as shown in Figure 19 and Figure 20.

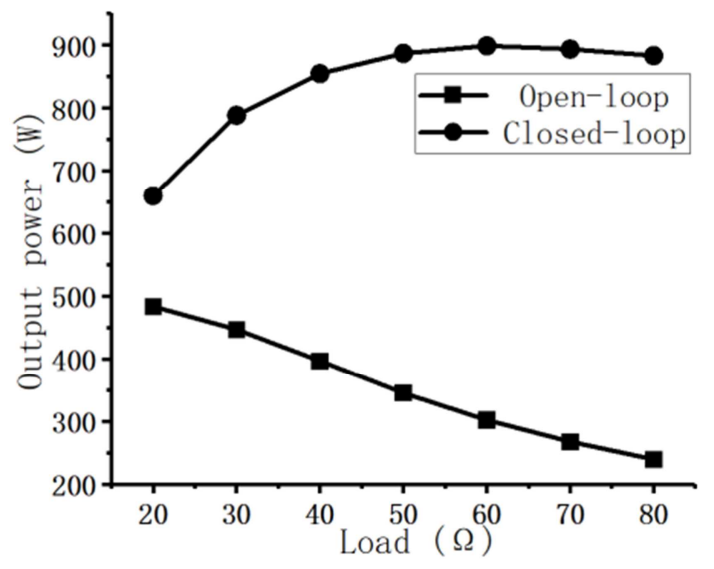

Figure 19. Relation of output power and load.

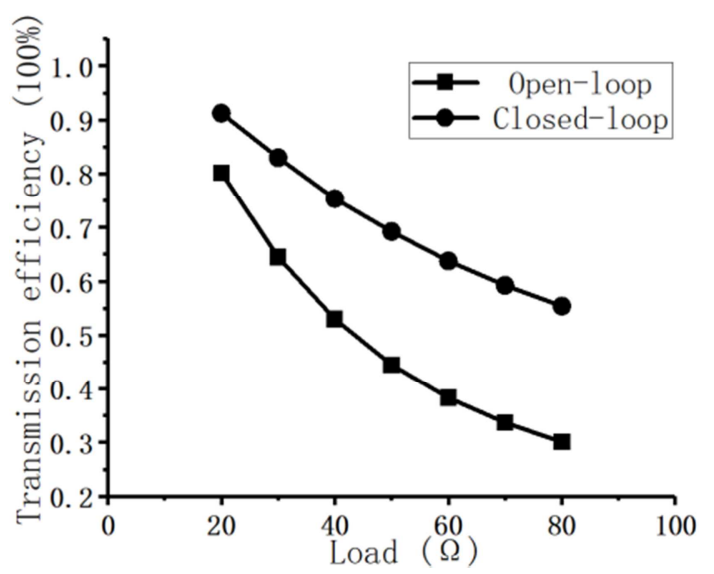

Figure 20. Relation of transmission efficiency and load.

As can be seen above, with the change of mutual inductance and load, the output power and transmission efficiency of the system are greatly improved by adopting CGI-PLL control algorithm when the primary coil is out of resonance and the secondary coil is in resonance.

\section{Conclusion}

Based on paper [17], this paper adopts a new control algorithm - CGI-PLL [18], is used to control the detuning of wireless energy transmission system when the primary coil is detuning and the secondary coil is resonant. By using CGI-PLL control algorithm, the active frequency tracking can be realized, and the phase angle between the output voltage and current of the inverter can be controlled by controlling the input phase angle difference parameter. The results show that the method can provide accurate phase compensation for the system, and the output power and transmission efficiency can be significantly improved by using the control algorithm.

\section{Acknowledgements}

This work was supported by National Natural Science Foundation of China (51667005) and Guangxi Natural Science Foundation (2018GXNSFDA281037). The authors would like to thank the associate editor and the experts of the Conference for their constructive suggestions.

\section{References}

[1] Dang Z, Yuan C, Qahouq J A A. Reconfigurable Magnetic Resonance-Coupled Wireless Power Transfer System [J]. IEEE Transactions on Power Electronics, 2015, 30 (11): 6057-6069.

[2] Hata K, Huang X, Hori Y. Power flow control of magnetic resonance wireless charing for hybrid energy storage system of electric vehicles application [C]// Society of Instrument \& Control Engineers of Japan. 2015.

[3] Wang Y, Song J, Lin L, et al. Research on magnetic coupling resonance wireless power transfer system with variable coil structure $[\mathrm{C}] / /$ Emerging Technologies: Wireless Power Transfer. 2017.

[4] Tian J, Hu A P. A DC-Voltage-Controlled Variable Capacitor for Stabilizing the ZVS Frequency of a Resonant Converter for Wireless Power Transfer [J]. IEEE Transactions on Power Electronics, 2017, 32 (3): 2312-2318.

[5] Masood Moghaddami, Aditya Sundararajan, Arif 1. Sarwat A. Power-Frequency Controller With Resonance Frequency Tracking Capability for Inductive Power Transfer Systems [J]. IEEE Transactions on Industry Applications, 2018, 54 (2): 1773-1783.

[6] Kim N Y, Kim K Y, Kim C W. Automated frequency tracking system for efficient mid - range magnetic resonance wireless power transfer $[\mathrm{J}]$. Microwave and Optical Technology Letters, 2012, 54 (6): 1423-1426.

[7] Dong X, Yin S, Wang D. Analysis of frequency splitting phenomena for magnetic resonance wireless power transfer systems [C] // 2018.

[8] Merugu Kavitha, Phaneendra Babu Bobba, Dinkar Prasad. Effect of coil geometry and shielding on wireless power transfer system [C]// 2016 IEEE 7th Power India International Conference (PIICON). IEEE, 2016.

[9] Qi Jiang, Yuannian Qin, Yubin Zhao etal. A receiver position estimation scheme in wireless power transfer system [C]// 2018 IEEE International Conference on Consumer Electronics (ICCE). IEEE, 2018. 
[10] Cheng Z, Y. Lu, Liu Q, etal. Research of Adaptive Tuning Magnetically Coupled Resonant Wireless Power Transfer System Based on Magnetic Amplifier [J]. Diangong Jishu Xuebao / Transactions of China Electrotechnical Society, 2018, 33: 305-312.

[11] Lim Y, Tang H, Lim S, etal. An adaptive impedance-matching network based on a novel capacitor matrix for wireless power transfer [J]. Power Electronics, IEEE Transactions on, 2014, 29 (8): 4403-4413.

[12] Zhao Y, Li X, Xu C Z, etal. Adaptive random beamforming for MIMO wireless power transfer system [C] // 2018 IEEE Wireless Communications and Networking Conference (WCNC). 2018.

[13] Lee J, Lim Y S, Yang W J, etal. Wireless Power Transfer System Adaptive to Change in Coil Separation [J]. IEEE Transactions on Antennas \& Propagation, 2014, 62 (2): 889-897.

[14] Dai X, Sun Y. An Accurate Frequency Tracking Method Based on Short Current Detection for Inductive Power Transfer System [J]. IEEE Transactions on Industrial Electronics, 2014, 61 (2): 776-783.

[15] Han Y, Luo M, Zhao X, et al. Comparative Performance Evaluation of Orthogonal-Signal-Generators based Single-Phase PLL Algorithms [J]. IEEE Transactions on Power Electronics, 2015, 31 (5): 3932-3944.

[16] Guan Qingxin, Zhang Yu, Li Minying. Single phase phase phase-locked loop method based on differential link [J] Chinese Journal of electrical engineering, 2016, 36 (19): 5318-5325.

[17] Tan P A, He H, Gao X. A Frequency-Tracking Method Based on a SOGI-PLL for Wireless Power Transfer Systems to Assure Operation in the Resonant State [J]. Journal of power electronics, 2016, 16 (3): 1056-1066.

[18] Kulkarni A, John V. Design of a fast response time single-phase PLL with dc offset rejection capability [J]. Electric Power Systems Research, 2017, 145: 35-43. 